\title{
Update: MIRIAM Registry and SBO
}

\author{
Nick Juty, EMBL-EBI \\ $3^{\text {rd }}$ Sept, 2011
}




\section{MIRIAM Registry}

- MIRIAM Guidelines ..

- MIRIAM Registry

- content

- URIs (URN form), example

- Summary/current developments

\section{SBO}

- Purpose and origins

- Term information stored

- Summary of changes since Edinburgh 


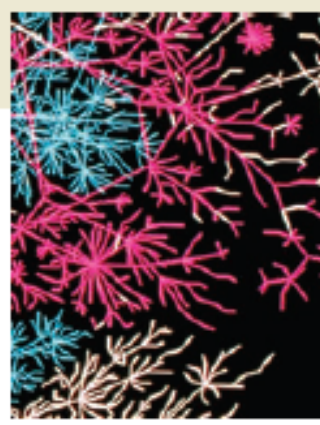

\section{Minimum information requested in the annotation of biochemical models (MIRIAM)}

Nicolas Le Novère ${ }^{1,15}$, Andrew Finney ${ }^{2,15}$, Michael Hucka ${ }^{3}$, Upinder S Bhalla ${ }^{4}$, Fabien Campagne ${ }^{5}$, Julio Collado-Vides ${ }^{6}$, Edmund J Crampin ${ }^{7}$, Matt Halstead ${ }^{7}$, Edda Klipp ${ }^{8}$, Pedro Mendes ${ }^{9}$, Poul Nielsen ${ }^{7}$, Herbert Sauro ${ }^{10}$, Bruce Shapiro ${ }^{11}$, Jacky L Snoep ${ }^{12}$, Hugh D Spence ${ }^{13}$ \& Barry L Wanner ${ }^{14}$

Most of the published quantitative models in biology are lost for the community because they are either not made available or they are insufficiently characterized to allow them to be reused. The lack of a standard description format, lack of stringent reviewing and authors' carelessness are

on the main causes for incomplete model descriptions. With ․ today's increased interest in detailed biochemical models, it is necessary to define a minimum quality standard for F the encoding of those models. We propose a set of rules for curating quantitative models of biological systems. These rules define procedures for encoding and annotating models $\cap$ represented in machine-readable form. We believe their
During the genomic era we have witnessed a vast increase in availability of large amounts of quantitative data. This is motivating a shift in the focus of molecular and cellular research from qualitative descriptions of biochemical interactions towards the quantification of such interactions and their dynamics. One of the tenets of systems biology is the use of quantitative models (see Box 1 for definitions) as a mechanism for capturing precise hypotheses and making predictions ${ }^{1,2}$. Many specialized models exist that attempt to explain aspects of the cellular machinery. However, as has happened with other types of biological information, such as sequences, macromolecular structures or 
Models must :

- be encoded in a public machine-readable format, standard compliant

- be named and clearly linked to a single reference description

- distribution terms

- contain creator's contact details

- reflect the structure of the biological processes described in the reference paper (list of reactions etc.)

- be instantiable in a simulation (possess initial conditions etc.)

- be able to reproduce the results given in the reference paper

- annotation to unambiguously identify each model constituent ... 


\section{MIRIAM guidelines for annotations}

\section{Annotation specification:}

- unambiguously relate component to external resource

- be encoded in a defined triplet format

- as a URI

- identifier unique for the data type

- optional use of qualifiers to refine relationship

- use a defined set of data types - community agreed

- approved data types stored in MIRIAM Registry 
- A data type is a set of data within which all data points refer to comparable entities, in terms of the 'properties' they can/do possess

- Individual data points (entities/records) share a common identifier scheme which can be used to distinguish them

- Data of the same 'kind'

- UniProt - protein data

- Chebi - small molecules

- PubMed - publication records 


\section{- Open access}

Anybody can access any public data without restriction (no commercial licence; no login page etc.)

\section{- Atomicity}

The granularity of the data distributed has to be appropriately selected (A database of "reactions" distributes reactions and not pathways) and consistent (e.g. classes or instances but not classes AND instances)

- Identifier

An atomic data is associated to a unique and perennial identifier

\section{- Community recognition}

The resource has to be "recognised" by the corresponding experimental community, be reasonably supported etc 


\section{EMBL-EBI}

\section{MIRIAM Registry}

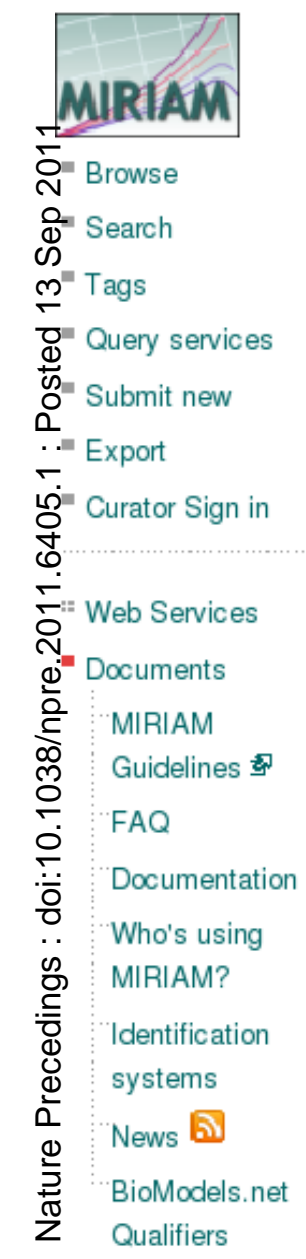

- MIRIAM on

SourceForge
EBI > Groups > Computational Neurobiology " Research > MIRIAM Registry

\section{MIRIAM Registry}

MIRIAM Registry are a set of online services created in support of MIRIAM, a set of guidelines for the annotation and curation of computational models.

The core of MIRIAM Registry is a catalogue of data types (namespaces corresponding to controlled vœabularies or databases), their URIs and the corresponding physical URLs or resources. Access to this data is made available via exports (XML) and Web Services (SOAP).

\section{MIRIAM}

\section{Quick links}

\begin{tabular}{|l|r|}
\hline $\begin{array}{l}\text { Browse } \\
\frac{\text { by data type name }}{\text { by tags }}\end{array}$ & Web Services \\
\hline Search & $\begin{array}{r}\frac{\text { services available }}{\text { usage of the services }} \\
\frac{\text { online demonstration }}{\text { Eeneric search }}\end{array}$ \\
\hline
\end{tabular}

\section{Registry}

MIRIAM Registry is composed of four components: a database, some Web Services, a Java library and this web application. 


\section{EMBL-EBI}

\section{MIRIAM Registry}

\begin{tabular}{ll}
\hline MIRIAM \\
\hline N
\end{tabular}

- MIRIAM on

SourceForge
EBI > Groups > Computational Neurobiology " Research ' MIRIAM Registry

\section{MIRIAM Registry}

MIRIAM Registry are a set of online services created in support of MIRIAM, a set of guidelines for the annotation and curation of computational models.

The core of MIRIAM Registry is a catalogue of data types (namespaces corresponding to controlled vœabularies or databases), their URIs and the corresponding physical URLs or resources. Access to this data is made available via exports (XML) and Web Services (SOAP).

MIRAAM

MIRIAM Registry is developed and maintained under the BioModels. net siv $^{3}$ initiative, and are free for use by all.

Quick links

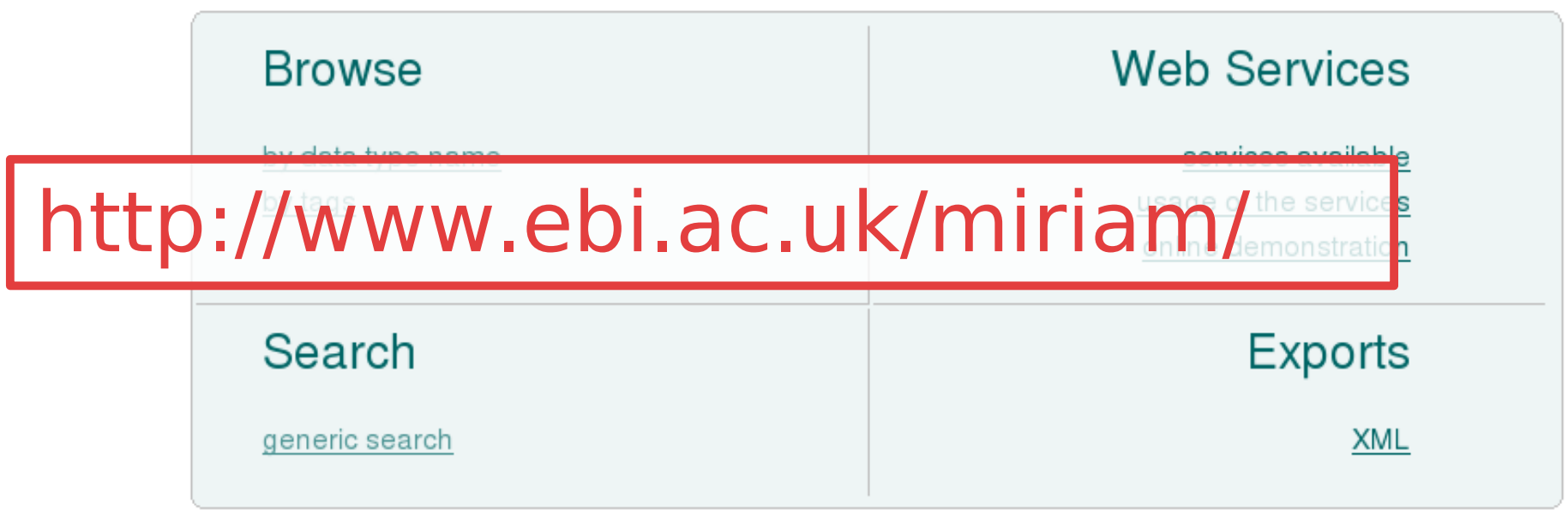

Registry

MIRIAM Registry is composed of four components: a database, some Web Services, a Java library and this web application.

COMBINE, Heidelberg, Germany 3-7 ${ }^{\text {th }}$ Sept, 2011 


\section{MIRIAM}

" Browse

흘 Search

응 Tags

क्. Query services

$\stackrel{m}{-}$

등 Submit new

$\frac{d}{\omega}$ Export

" Curator Sign in

$\because$

영 Web Services

Q, Documents

둥 MIRIAM

¿ Guidelines

흘 $\mathrm{FAQ}$

Documentation

Who's using

MIRIAM?

Identification

systems

News 1

BioModels.net

Qualifiers

$\stackrel{0}{=}$

荧 MIRIAM on

$Z$ SourceForge

\section{" Support}

- Contact
$\mathrm{EBI}$ > Groups > Computational Neurobiology > Research > MIRIAM Registry

Browse data types: recently updated

Recently updated $|\underline{A}| \underline{B}|\underline{C}| \underline{D}|\underline{E}| \underline{F}|\underline{G}| \underline{H}|\underline{l}| \underline{J}|\underline{K}| \underline{L}|\underline{M}| \underline{N}|\underline{O}| \underline{P}|\underline{Q}| \underline{R}|\underline{s}| \underline{T}|\underline{\cup}| \underline{\mathrm{V}}|\underline{\mathrm{W}}| \underline{\mathrm{X}}|\underline{\mathrm{Y}}| \underline{\mathrm{Z}}$

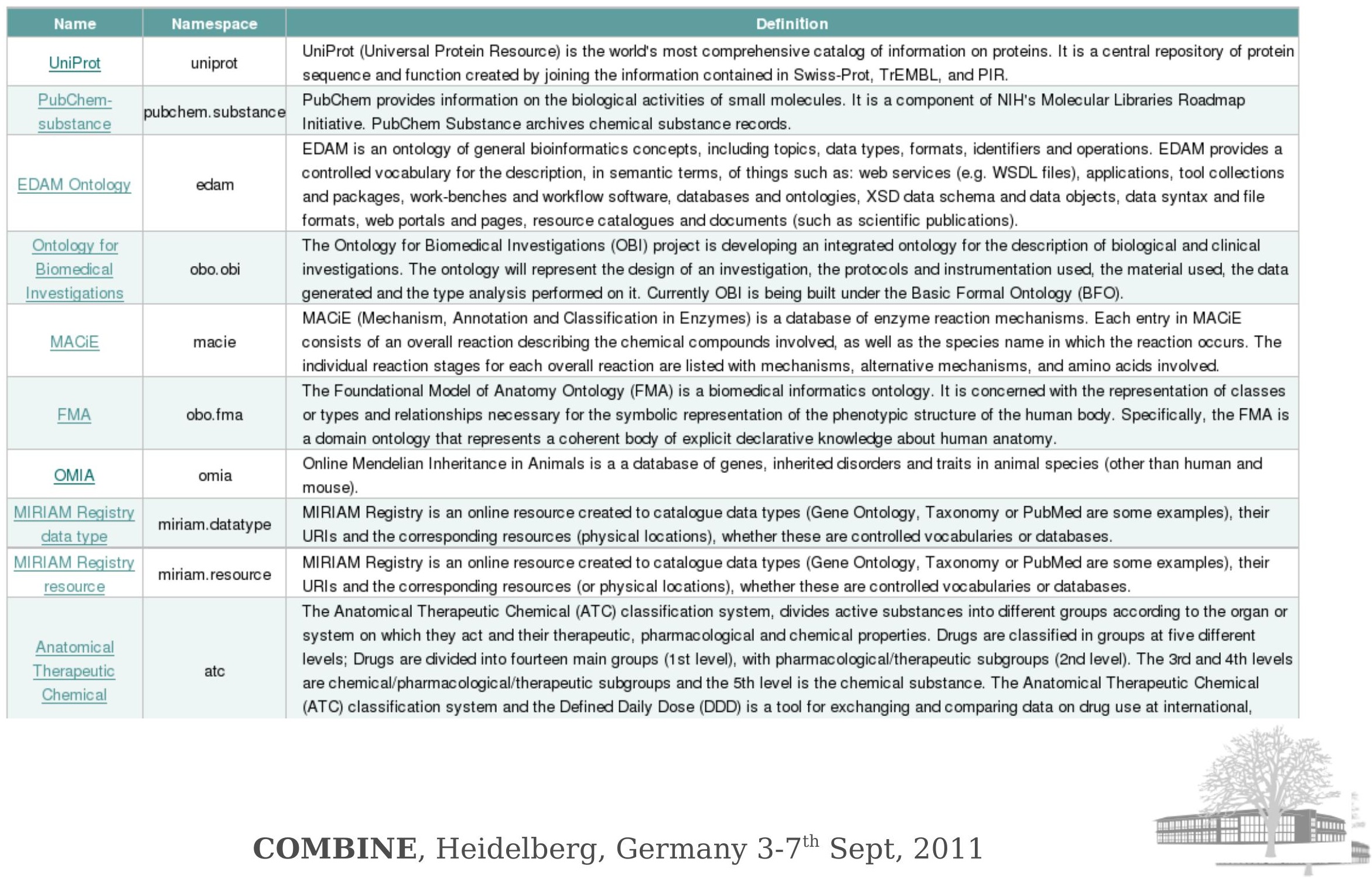


Data type: Enzyme Nomenclature

\begin{tabular}{|c|c|c|c|}
\hline General & Tags & Example Usage & Web Services \\
\hline \multicolumn{4}{|c|}{ General information about the data type } \\
\hline \multicolumn{4}{|r|}{ Name } \\
\hline \multicolumn{3}{|l|}{ Identifier } & MIR:00000004 \\
\hline \multicolumn{3}{|l|}{ Name } & Enzyme Nomenclature \\
\hline \multirow{3}{*}{\multicolumn{3}{|c|}{ Synonyms }} & EC code \\
\hline & & & Enzyme Classification \\
\hline & & & EC \\
\hline \multicolumn{4}{|r|}{ URls } \\
\hline \multicolumn{3}{|c|}{ MIRIAM URN } & urn:miriam:ec-code \\
\hline \multirow{3}{*}{\multicolumn{3}{|c|}{ Deprecated }} & http://www.ec-code.org/ \\
\hline & & & urn:Isid:ec-code.org \\
\hline & & & http://www.ebi.ac.uk/IntEnz/ \\
\hline \multicolumn{4}{|r|}{ Information _ } \\
\hline \multicolumn{3}{|l|}{ Definition } & $\begin{array}{l}\text { The Enzyme Classification contains the recommendations of the Nomenclature Committee of the International Union of Biochemistry and Molecular } \\
\text { Biology on the nomenclature and classification of enzyme-catalysed reactions. }\end{array}$ \\
\hline & $\wedge \cdot d+1 .-1 .-1 .-1 \cdot d+1.1 d+1 .-1 .-1 \cdot d+1.1 d+1.1 d+1 .-1 \cdot d+1.1 d+1.1 d+1.1 d+\$$ \\
\hline \multicolumn{3}{|c|}{ Identifier Pattern } & Physical Locations \\
\hline \multirow{4}{*}{\multicolumn{2}{|c|}{$\begin{array}{c}\text { Resource } \\
\text { MIR:00100002 }\end{array}$}} & Access URL & http://www.genome.jp/dbget-bin/www_bget?ec:\$id [Example: 1.1.1.1 溦] \\
\hline & & Website & http://www.genome.jp/dbget-bin/www_bfind?enzyme \\
\hline & & Description & KEGG Ligand Database for Enzyme Nomenclature \\
\hline & & Institution & Kyoto University Bioinformatics Center, Japan \\
\hline \multirow{4}{*}{\multicolumn{2}{|c|}{$\begin{array}{c}\text { Resource } \\
\text { MIR:00100003 }\end{array}$}} & Access URL & http://us.expasy.org/cgi-bin/nicezyme.pl?\$id [Example: 1.1.1.1 眍] \\
\hline & & Website & $\underline{\text { http://us.expasy.org/enzyme/ }}$ \\
\hline & & Description & Enzyme nomenclature database, ExPASy (Expert Protein Analysis System) \\
\hline & & Institution & Swiss Institute of Bioinformatics, Switzerland \\
\hline \multirow{4}{*}{\multicolumn{2}{|c|}{$\begin{array}{c}\text { Resource } \\
\text { MIR:00100001 }\end{array}$}} & Access URL & http://www.ebi.ac.uk/intenz/query?cmd=SearchEC\&ec=\$id [Example: 1.1.1.1 蹸] \\
\hline & & Website & $\underline{\text { http://www.ebi.ac.uk/intenz/ }}$ \\
\hline & & Description & IntEnZ (Integrated relational Enzyme database) \\
\hline & & Institution & European Bioinformatics Institute, United Kingdom \\
\hline \multicolumn{4}{|r|}{ References } \\
\hline \multirow{2}{*}{\multicolumn{2}{|c|}{ URL(s) }} & & http://www.chem.qmul.ac.uk/iubmb/enzyme/ \\
\hline & & & 田 http://srs.ebi.ac.uk/srsbin/cgi-bin/wgetz?-view+MedlineFull+[medline-PMID:10812475] \\
\hline \multicolumn{4}{|r|}{ Miscellaneous } \\
\hline \multicolumn{3}{|c|}{ Date of creation } & 2006-08-14 19:38:06 GMT \\
\hline \multicolumn{3}{|c|}{ Date of last modification } & 2010-12-09 08:45:47 GMT \\
\hline
\end{tabular}

COMBINE, Heidelberg, Germany 3-7 $7^{\text {th }}$ Sept, 2011 


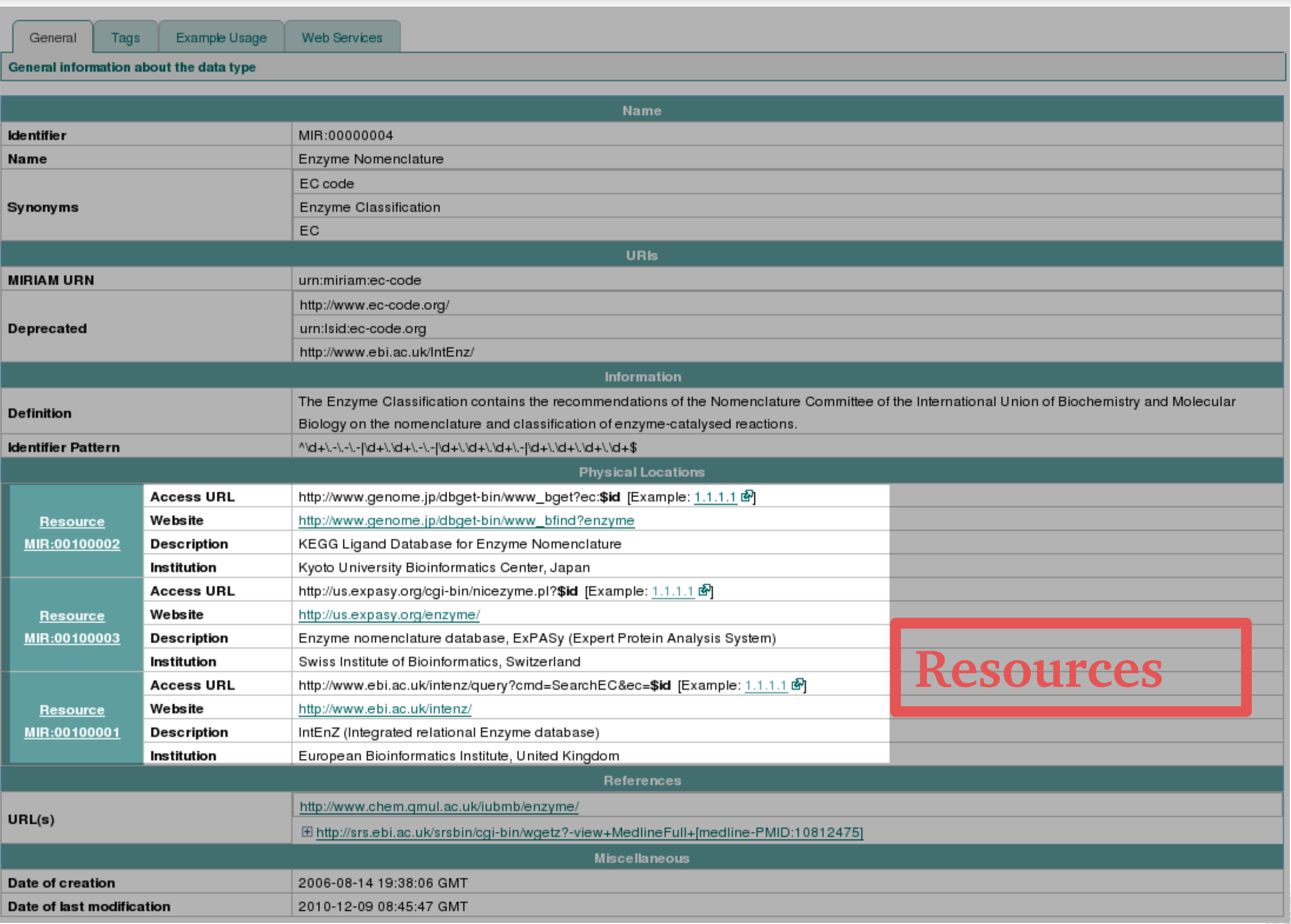

COMBINE, Heidelberg, Germany 3-7 $7^{\text {th }}$ Sept, 2011 


\section{EMBL-EBI}

\section{Resource monitoring}

\section{Resource: MIR:00100050}

_ General information about the resource: The FlyBase Database (associated with the data type: FlyBase).

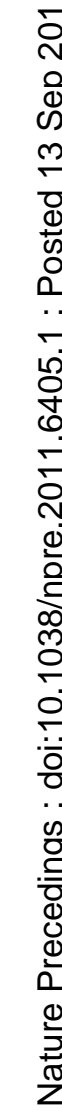

\begin{tabular}{|c|c|}
\hline & Health s \\
\hline Last known state & up \\
\hline Last check & 2011-03-29 06:37:48 \\
\hline Uptime ratio & $100 \%$ (763 checks) \\
\hline Downtime ratio & $0 \%(0$ checks $)$ \\
\hline Unknown ratio & $0 \%(0$ checks $)$ \\
\hline URL used & http://www.flybase.org/reports/FBgn0011293.html 通 \\
\hline
\end{tabular}

Lั?

Health history

Full record of the health checks performed on this resource.

2011

읃

January

February

March

ō April

May

ก) June

Ō July

August

September

() October

November

December

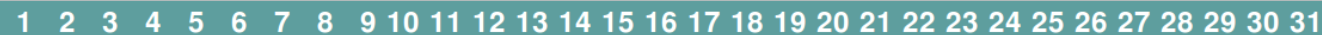

2010

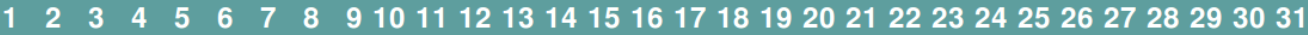

\begin{tabular}{|l|}
\hline January \\
\hline February \\
\hline March \\
\hline April \\
\hline
\end{tabular}

COMBINE, Heidelberg, Germany 3-7 ${ }^{\text {th }}$ Sept, 2011

Legend

up and responsive probably up resource down state unknown no data available 


\section{EMBL-EBI}

\section{Resource monitoring}

nesource: IvIr:UUIUUU42

WARNING! This resource has been deprecated!

Please use the other resources providing the same dataset

General information about the resource: WormBase Mirror at Marseille-Nice Genopole (associated with the data type: WormBase).

\begin{tabular}{|c|c|}
\hline & Health statistics \\
\hline Last known state & down \\
\hline Last check & 2010-09-15 09:11:48 \\
\hline Uptime ratio & $43 \%$ ( 245 checks) \\
\hline Downtime ratio & $56 \%$ (318 checks) \\
\hline Unknown ratio & $0 \%(0$ checks $)$ \\
\hline URL used & http://crfb-3.univ-mrs.fr/db/gene/gene?name=WBGene00000001;class=Gene $\mathbf{s}^{*}$ \\
\hline
\end{tabular}

\section{Health history}

Full record of the health checks performed on this resource.

2010

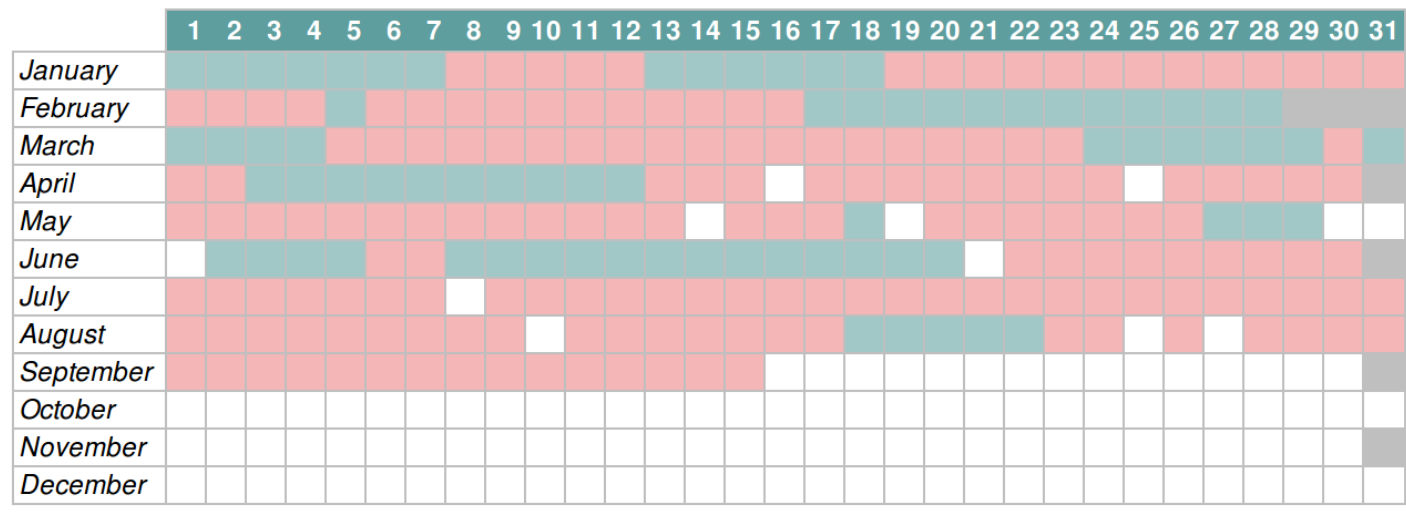

2009

\section{Legend}

up and responsive probably up resource down state unknown no data available

COMBINE, Heidelberg, Germany 3-7 th $^{\text {Sept, }} 2011$

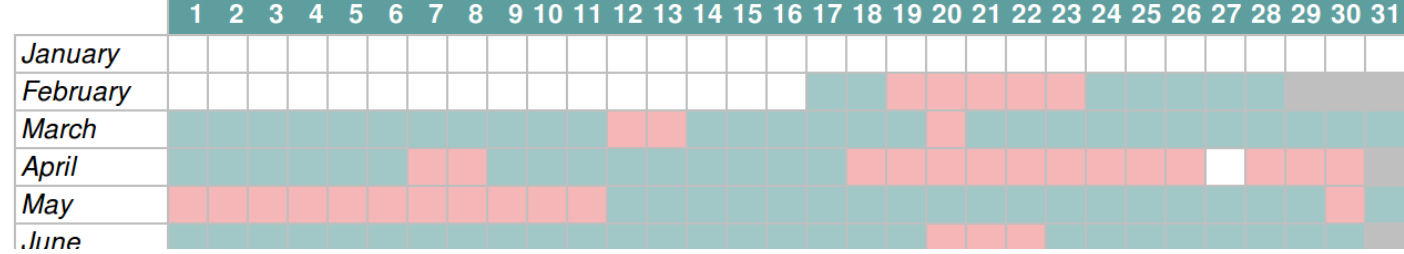




\section{EMBL-EBI}

\section{Registry submissions}

\section{Add a data type}

Please fill this form in order to submit a new data type to MIRIAM Resources. Alternatively, you can contact us with your query.

The new data type will not be directly publicly available after you pressed the Submit button. A curator will first check if it complies with the terms and conditions of MIRIAM Resources before publishing it.

\section{Help}

You can display all help bubbles by clicking on: Displays all the help messages, or hide them: Hides all the help messages.

Moreover, you can display the individual help by clicking on the button: located in the title of each section.

\section{Add a new data type}

First you need to enter the name of the data type you want to add to the database. After you can add as much synonyms as you want.

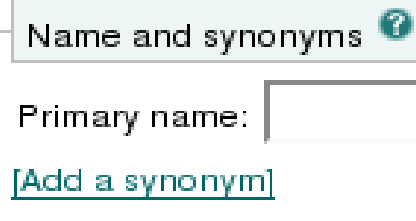

Here is some information about the data type: definition and regular expression (i.e. pattern for identifiers of elements, following the PERL style).

\begin{tabular}{l|l|}
\hline Definition and pattern 8 \\
Definition: \\
Identifier pattern:
\end{tabular}

COMBINE, Heidelberg, Germany 3-7 ${ }^{\text {th }}$ Sept, 2011 


\section{- MIRIAM Database}

data type catalogue

MIRIAM Web Services

programming interface for querying database

\section{MIRIAM Library}

ready to use Java code

\section{MIRIAM Web Site}

- web page for browsing and querying

- allows new submission (curated)

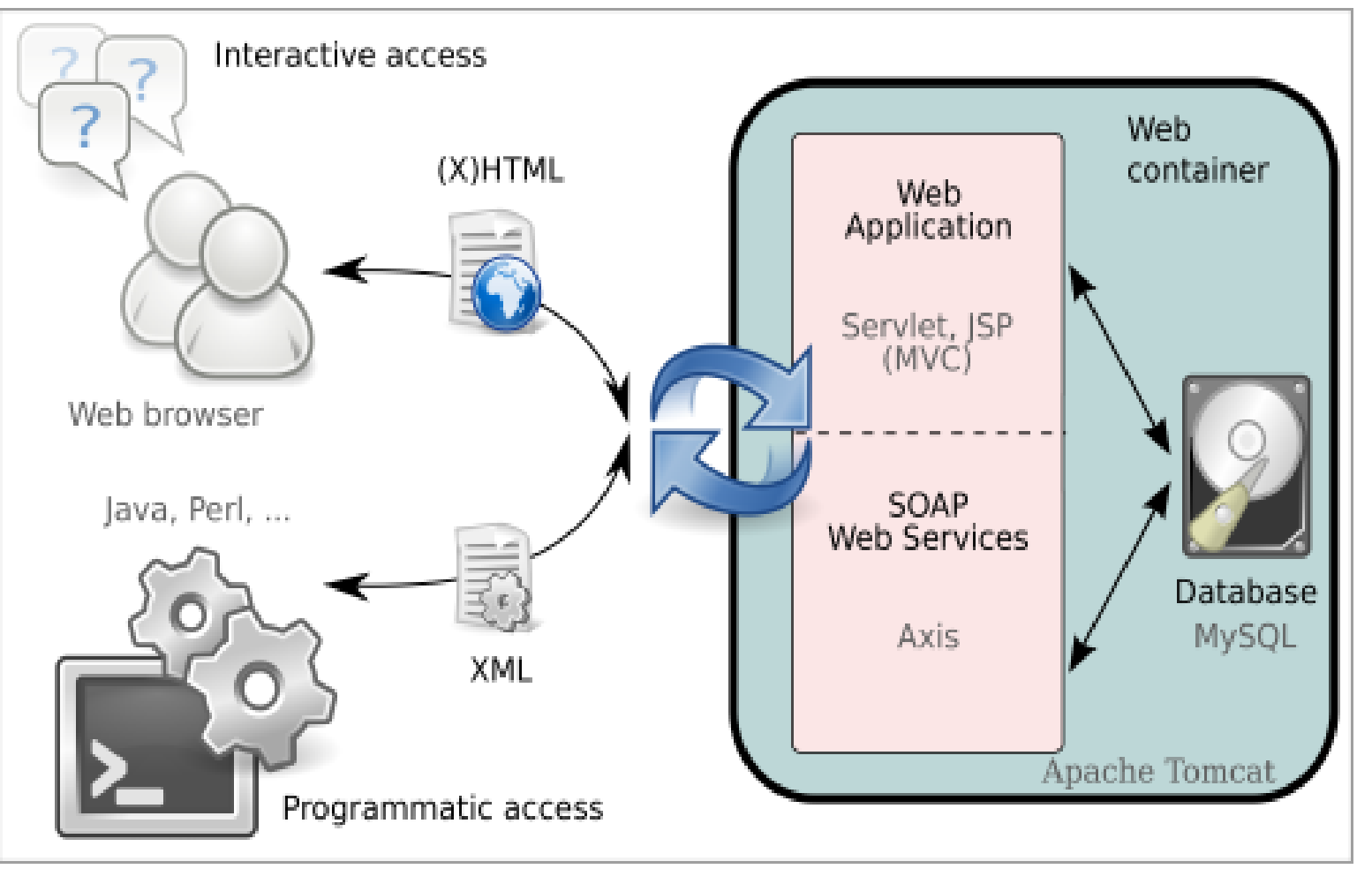

Laibe and Le Novère.

MIRIAM Resources: tools to generate and resolve robust cross-references in Systems Biology.

BMC Systems Biology, 2007 


\section{EMBL-EBI}

\section{Web Services: generate URI}

\section{Web Services Demonstration}

F Here is a Web interface which allows you to perform some queries on MIRIAM Database.

尺

잉 These queries are an example of what MIRIAM Web Services can provide you.

For more information, please refer to the complete list of available queries.

感

Query

เి

Choose one action from the list: get the MIRIAM URI of an element $\quad$ Go!

흘 Get the MIRIAM URI of an element or entity:

ò

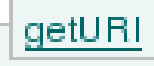

urn:miriam:pubmed:18078503

COMBINE, Heidelberg, Germany 3-7 ${ }^{\text {th }}$ Sept, 2011 


\section{EMBL-EBI}

\section{Web Services Demonstration}

$\mp$ Here is a Web interface which allows you to perform some queries on MIRIAM Database.

ก

$\stackrel{\circ}{\mathscr{N}}$ These queries are an example of what MIRIAM Web Services can provide you.

For more information, please refer to the complete list of available queries.

¿

Query

웅

Choose one action from the list:

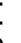

Get links to access an element:

getLocations

MIRIAM URI Um: miriam:pubmed: 18078503

Search

\section{Answer}

帚

n http://www.ncbi.nlm.nih.gov/pubmed/18078503

n http://srs.ebi.ac.uk/srsbin/cqi-bin/wgetz?-view+MedlineFull+[medline-PMID:18078503]

- http://www.ebi.ac.uk/citexplore/citationDetails.do?dataSource=MED\&externalld=18078503

- http://www.hubmed.org/display.cgi?uids $=18078503$

COMBINE, Heidelberg, Germany 3-7 th $^{\text {Sept, }} 2011$ 
Data type identifier

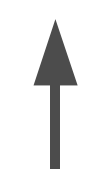

scheme identifier
Data-set Identifier

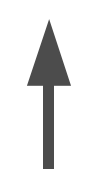

text string

\section{Annotation qualifier}

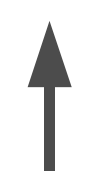

optional text string

(MIRIAM publication)

(Caffeine)

- Qualifiers are a Controlled vocabulary (CV)

- CV established by the community

- http://biomodels.net/qualifiers/ 


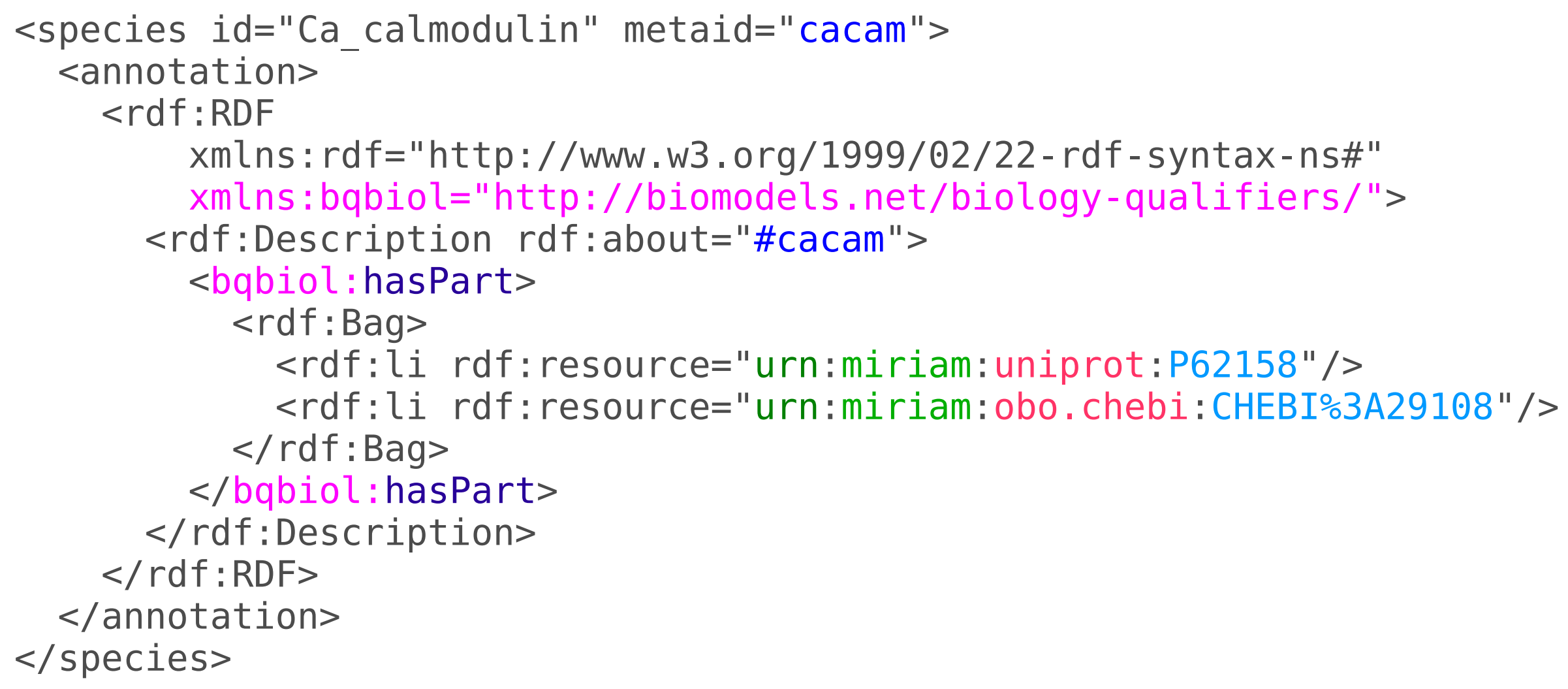

cacam hasPart P62158 and CHEBI:29108

\section{model component relationship external resource(s)}


$\bar{\sigma}^{-}$Introduction of a URL scheme, in addition to the URN namespace

- In discussions with Bio2RDF

Collaboration with BioDbCore

More structured tags

Growth in content/coverage Limited support 'branch' 


\title{
The Systems Biology Ontology
}

\author{
http : //biomodels . net/sbo
}

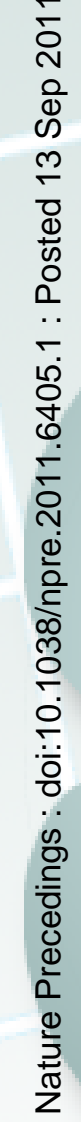




\section{http://www.obofoundry.org/}

\section{$\$$ The Open Biomedical Ontologies}
Ontologies
Resources
Participate
About

The $\mathrm{OBO}$ Foundry is a collaborative experiment involving developers of science-based ontologies who are establishing a set of principles for ontology development with the goal of creating a suite of orthogonal interoperable reference ontologies in the biomedical domain. The groups developing ontologies who have expressed an interest in this goal are listed below, followed by other relevant efforts in this domain.

In addition to a listing of $\mathrm{OBO}$ ontologies, this site also provides a statement of the $\mathrm{OBO}$ Foundry principles, discussion fora, technical infrastructure, and other sewices to facilitate ontology development. We welcome feedback and encourage participation.

Click any column header to sort the table by that column. The is link to the term request trackers for the listed ontologies.

\begin{tabular}{|c|c|c|c|c|}
\hline \multicolumn{5}{|c|}{ OBO Foundry candidate ontologies } \\
\hline Amphibian gross anatomy & anatomy & AAO & amphibian anatomy.obo & $2008108 / 19$ \\
\hline Ascomycete phenotype ontology & phenotype & APO & ascomplote phenotype.obo & $200907 / 10$ \\
\hline Biological process & biobgical process & 90 & gene ontology edit.obo t: & $200908 / 21$ \\
\hline C. elegans phenotype & phenotype & WBPhenotype & worm phenotype.obo & $200808 / 19$ \\
\hline ح-11+..- & -..._-_. & $\wedge$ & 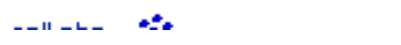 & manounmon \\
\hline
\end{tabular}




\section{The Systems Biology Ontology http://biomodels.net/sbo}

- A navigable taxonomic structure of terms that has 'parents', 'children'

- Provide a strictly defined relational vocabulary of terms for use in Computational Biology

- Describe model components and how they are intended to be used

- 7 orthogonal vocabularies containing domain knowledge:

- type of entity (compartment / macromolecule)

- roles of entities (modifier / product)

- how they interact (reaction / transport) 


\section{SBO Tree}

$\boxplus \square$

$\bar{\sigma}^{\square} \underline{\text { SBO:0000000 - systems biology representation }}$
I) (I) SBO:0000355 - conservation law
t) (1) SBO:0000474 - convenience function
$\square$ (c) SBO:0000001 - rate law

$\square(\mathbf{I}$ SBO:0000268 - enzymatic rate law

$\boxminus(1) \underline{\text { SBO:0000150 - enzymatic rate law for irreve }}$

(1) SBO:0000151 - enzymatic rate law fo

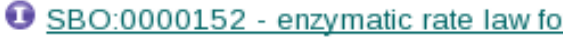

$\square$ (I) SBO:0000028 - enzymatic rate law for

(1) SBO:0000031 - Briggs-Hald:

(1) SBO:0000029 - Henri-Michaelis

(I) $\underline{\mathrm{SBO} 0000199 \text { - normalised enz }}$

(I) SBO:0000030 - Van Slyke-Cull

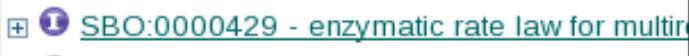

I) (1) SBO:0000269 - enzymatic rate law for unire

$\rightarrow(1)$ SBO:0000425 - reversible Hill-type enzymat

t(1) $\mathrm{SBO} 0000192$ - Hill-type rate law. generalised forn

(t) 1 SBO:0000012 - mass action rate law

t) (I) SBO:0000527 - modular rate law

(1) SBO:0000391 - steady state expression

(1) SBO:0000544 - metadata representation

†) 1 SBO:0000004 - modelling framework

II $\mathrm{SBO} 0000231$ occurring entity representation

t) (1) SBO:0000003-participant role

It (I) SBO:0000236 - physical entity representation

\#) $\mathrm{SBO} 0000545$ systems description parameter \begin{tabular}{|l|l|l|}
\hline (6) hystems Biology Ontology - Mozilla Firefox & htwww.ebi.ac.uk/sbo/main/browse.jsp?nodeld=32 \\
\hline
\end{tabular}

\section{Term: SBO:0000031}

Name

\section{Briggs-Haldane rate law}

Definition

The Briggs-Haldane rate law is a general rate equation that does not require the restriction of

equilibrium of Henri-Michaelis-Menten or irreversible reactions of Van Slyke, but instead make the

hypothesis that the complex enzyme-substrate is in quasi-steady-state. Although of the same form than

the Henri-Michaelis-Menten equation, it is semantically different since $\mathrm{Km}$ now represents a pseudo-

equilibrium constant, and is equal to the ratio between the rate of consumption of the complex (sum of

dissociation of substrate and generation of product) and the association rate of the enzyme and the substrate.

MathML

math xmlns="http://www .w3.org/1998/Hath/Hathll"

semantics definitionURL="http://biomodels .net/S1 O/\#SBO:0000062" >

$<1$ ambda $>$

bvar $><$ ci definitionURL="http://biomodels.net $/$ SBO/\#SBO:0000025" $>$ kcat $</$ ci $></$ bvar $>$

$\langle$ bvar $><$ ci definitionURL="http: //biomodels. net $/$ SBO $/$

$<$ bvar $><c i$ definitionURL="http: $/ /$ biomodels $\cdot$ net $/$ SBO $/$

$<$ bvar $><$ ci definitionURL="htt $: / /$ biomodels. net $/$ SBO

$<$ apply>

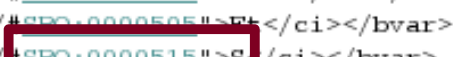

SBO 0.000310 S. $/$ ci $></$ bvar $>$ aimidar

Rendered equation

$\lambda(\mathrm{kcat}, \mathrm{Et}, S, \mathrm{Km})=\frac{\mathrm{kcat} \times \mathrm{Et} \times S}{\mathrm{Km}+S}$

Comment

Rate-law presented by G.E. Briggs and J.B.S. Haldane (1925): "A note on the kinetics of enzyme action, Biochem. J., 19: 338-339".

Miscellaneous

Date of creation:

23 February 2006, 14:00

Date of last modification:
05 February 2010, 10:48
Continuous framework

Parent(s)

SBO:0000028 enzymatic rate law for irreversible non-modulated non-interacting unireactant enzymes (is a) Children

This term has no child.

Entity

History [+]

\section{Quantitative parameter}

COMBINE, Heidelberg, Germany 3-7 $7^{\text {th }}$ Sept, 2011 
Semantic layer:

- conversion to semantically enriched computing formats (such as BioPAX)

- link between models encoded in SBML and graphical notations (such as SBGN)

- translation of models between continuous deterministic frameworks and discrete stochastic framework

- merging and integration of models 


\section{https://sourceforge.net/projects/sbo}

Systems Biology Ontology
\begin{tabular}{|l|l|l|l|l|l}
\hline Summary Files Reviews Support Develop Tracker Forums Code & Watch \\
\hline
\end{tabular}

- Add new Browse Reporting Admin

¿

웡 Tracker: Term request

$m$ List of suggested SBO term creations or modifications.

잉

$\frac{\Phi}{4}$

Search

Search

-

足

Page: Page 1 Next $\rightarrow$

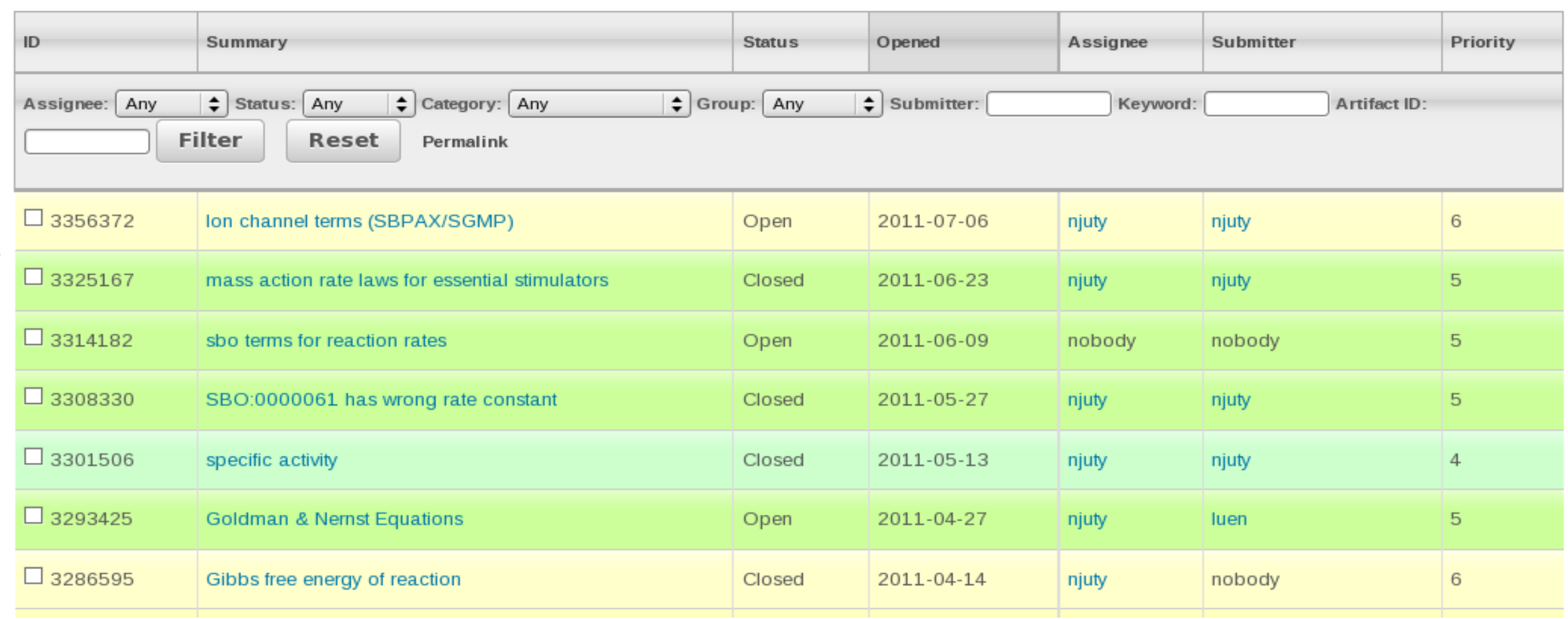

COMBINE, Heidelberg, Germany 3-7 $7^{\text {th }}$ Sept, 2011 
Problem: 'substrate' (participant role) = amount? concentration?

$<$ speciesReference>

$<$ parameter $>$

$<$ kineticLaw $>$

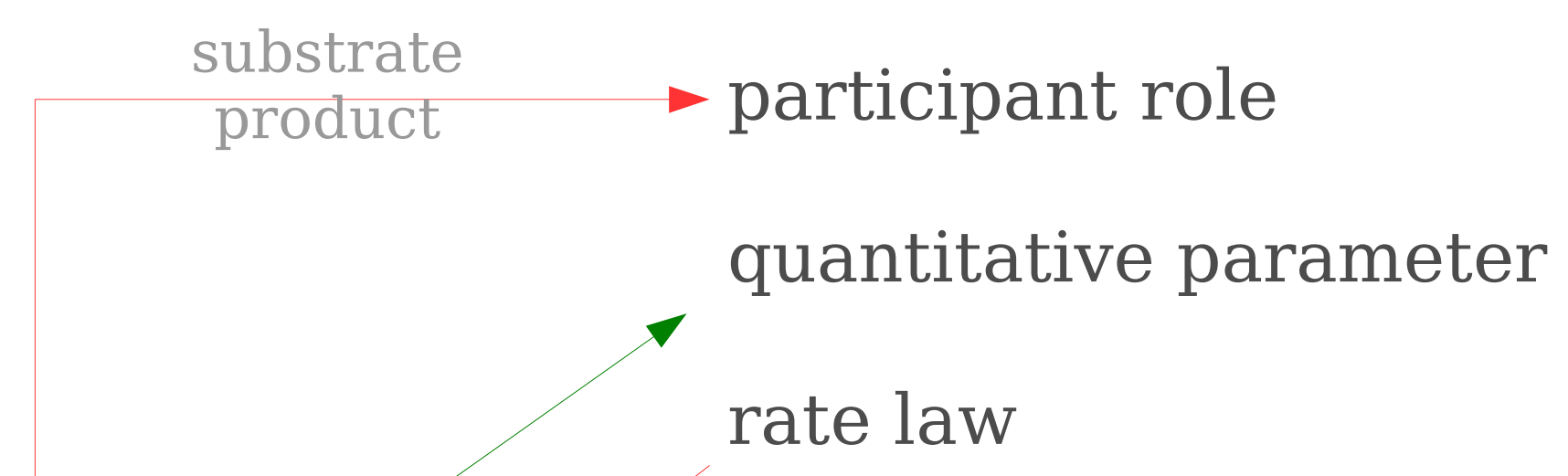

MathML

$<$ bvar>

SBO:0000015"> substrate <..

SBO:0000011"> product <.. 
- Initial changes made on SBO 'demo'

- Introduce 'quantitative parameter's for 'participant role' terms

\section{quantitative} parameter

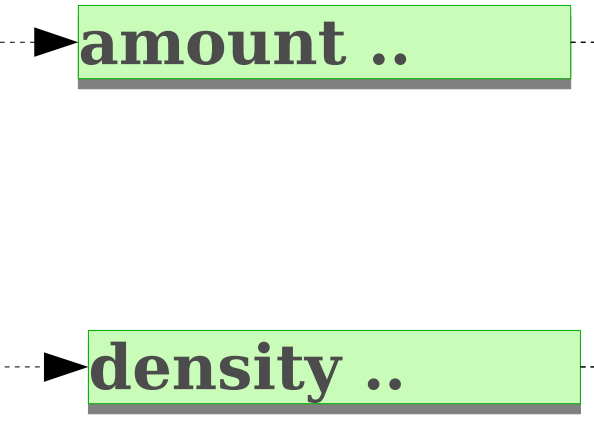

-number..

-concentration ..

- mass density ..

- Modify <bvar> links in all MathML - redirect to quantitiative parameter branches

- Request feedback for selected users (validate)

- Move changes to SBO 'main' (implement $\rightarrow$ live version) 
SBGN

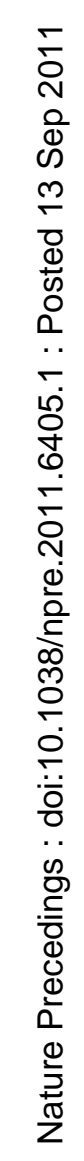

$<-$

Process

Stimulation

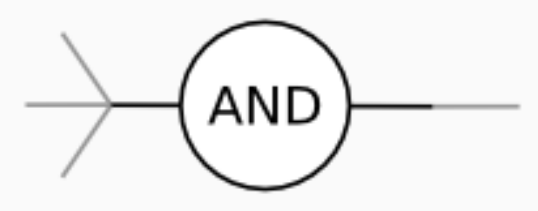

(PD)

(ER)

SBO

SBO:0000375

SBO:0000170

And (AF)
SBO:0000173 


\section{SBO / SBGN metadata}

\section{SBGN}

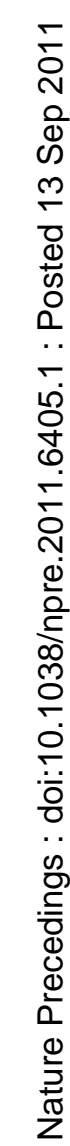

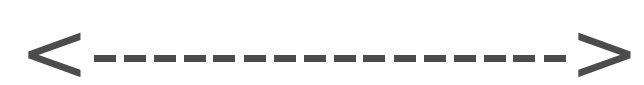

SBO

\section{Annotation (ER)}

- $7^{\text {th }}$ branch: Annotation, cross-references, metadata

${ }_{n}$ I| SBO:0000544 - metadata representation

II) SBO:0000550 - annotation

II S SBO:0000557 - embedded annotation

${ }_{7}(1)$ SBO:0000555 - controlled annotation

I SBO:0000551 - controlled short label

(1) SBO:0000556 - uncontrolled annotation

II I SBO:0000552 - reference annotation

(I) SBO:0000553 - bibliographical reference

(1) SBO:0000554 - database cross reference 
$\underline{\mathrm{SBO}+\mathrm{MIRIAM}}$

- Mélanie Courtot

- Camille Laibe

- Nicolas Le Novère

- Lukas Endler

$\underline{\text { SBML team }}$

- Michael Hucka

- Sarah Keating

BioModels Database

developers and curators

\section{The Systems Biology community for their contributions, software support and their comments.}

bbsrc

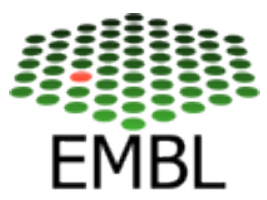




\section{Models}

Minimal

requirements

MIRIAM

implemented by

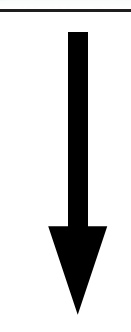

Example

Data-model

adds meaning to

Ontology
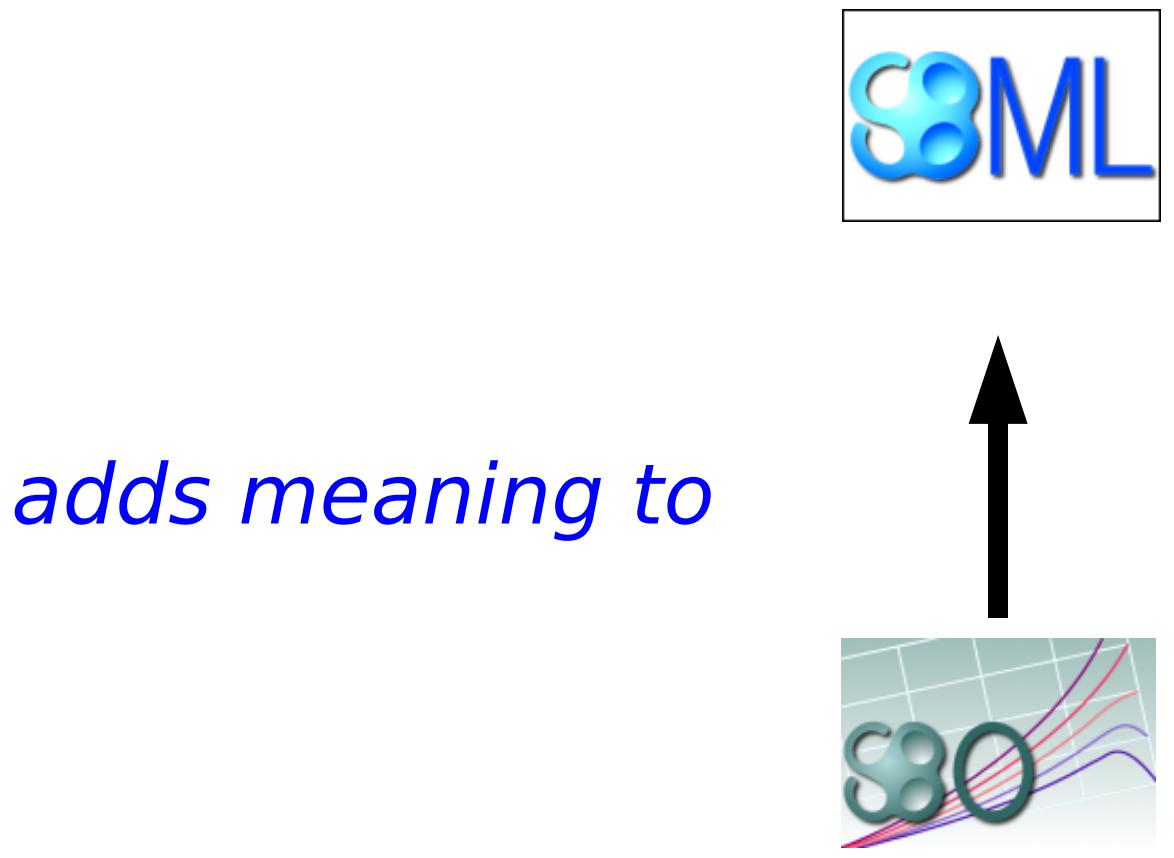


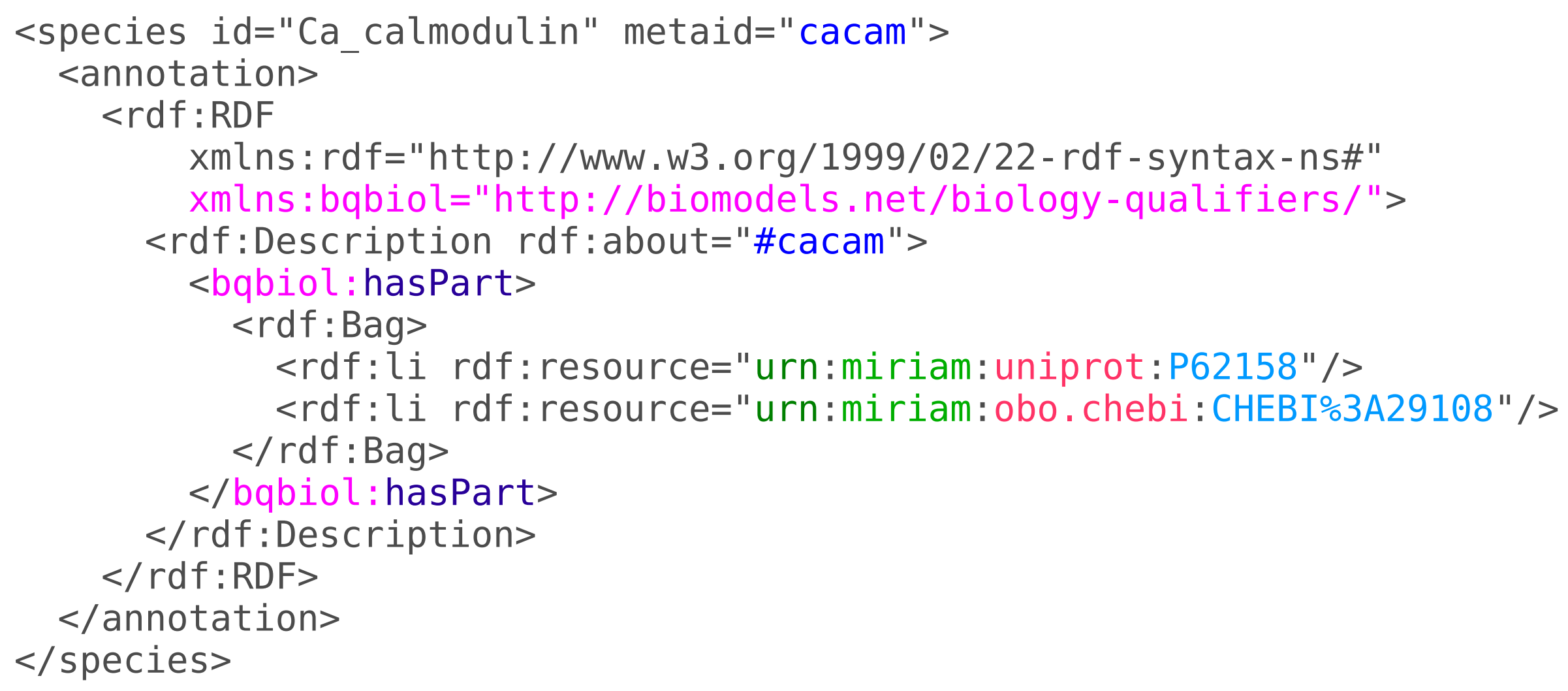




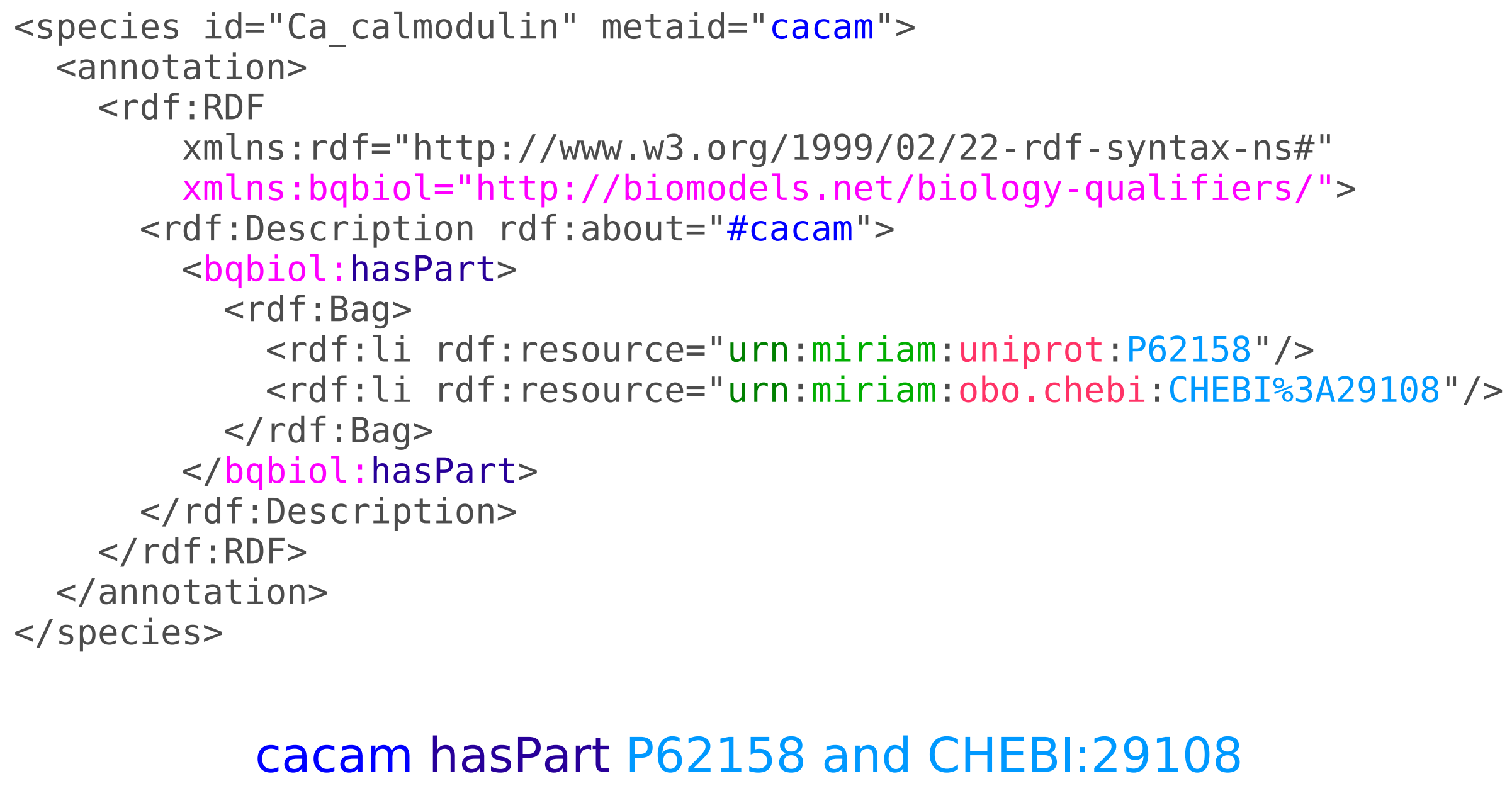

cacam hasPart P62158 and CHEBI:29108 